\title{
The Role of microRNA in
} Pathogenesis, Diagnosis, Different Variants, Treatment and Prognosis of Mycosis Fungoides

\author{
Pengfei Wen, Yao Xie and Lin Wang* \\ Department of Dermatovenerology, West China Hospital, Sichuan University, Chengdu, China
}

OPEN ACCESS

Edited by:

Pietro Quaglino,

University of Turin, Italy

Reviewed by:

Ivan V. Litvinov,

McGill University, Canada

Serena Bonin,

University of Trieste, Italy

Giusto Trevisan,

University of Trieste, Italy

${ }^{*}$ Correspondence:

Lin Wang

Ikzw|@126.com

Specialty section:

This article was submitted to

Skin Cancer,

a section of the journal

Frontiers in Oncology

Received: 03 August 2021 Accepted: 12 November 2021 Published: 13 December 2021

Citation:

Wen PF, Xie Y and Wang L (2021)

The Role of microRNA in

Pathogenesis, Diagnosis, Different

Variants, Treatment and Prognosis

of Mycosis Fungoides.

Front. Oncol. 11:752817.

doi: 10.3389/fonc.2021.752817
Mycosis fungoides (MF) is the most common type of cutaneous T-cell lymphoma (CTCL), accounting for approximately $50 \%$ of all CTCLs. Although various molecular changes in MF have been described in existing studies, no obvious disease-specific changes have been found thus far. microRNAs (miRs) are short, noncoding RNA molecules that play roles in the post-transcriptional regulation of oncogenes and tumor suppressor genes in various diseases. Recently, there has been rapidly expanding experimental evidence for the role of miRs in the progression, early diagnosis, prognosis prediction for MF. Efforts to improve early diagnosis and develop personalized therapy options have become more important in recent years. Here, we provide an overview and update of recent advances regarding miRs associated with MF. Furthermore, we provide insights into future opportunities for miR-based therapies.

Keywords: microRNA, Mycosis fungoides, pathogenesis, diagnosis, treatment, prognosis

\section{INTRODUCTION}

Mycosis fungoides (MF) is the most common type of cutaneous T-cell lymphoma (CTCL), accounting for approximately $50 \%$ of all CTCLs. The clinical manifestations usually present with erythematous patches and plaques with an indolent course and may slowly progress to tumors (1). Similar to inflammatory dermatosis, the lesions of MF patients in the stable stage can last for decades with a favorable prognosis. The period between the onset of skin lesions and diagnosis can vary from several months to years, with some patients' diagnoses delayed by more than four decades (2). However, in a proportion of early MF patients, the disease progresses rapidly and enters a more advanced stage with visceral spread, requiring more aggressive treatment regimens (3). Unfortunately, it is currently difficult to distinguish inflammatory dermatosis from early MF and identify patients with favorable or poor prognosis before treatment. Therefore, new approaches are needed to improve the accuracy of the early diagnosis and predict the prognosis of MF. 
Although various molecular changes in MF have been described in existing studies, including chromosomal, genomic, and gene expression aberrations; no obvious disease-specific changes have been found thus far (4). MF is a clonal disorder with specific $\mathrm{T}$-cell receptor (TCR) gene rearrangement. In addition to clinical and histological findings, TCR clonality testing is a helpful adjunct diagnostic method (5). However, the sensitivity of TCR rearrangement detection varies greatly according to different clinical stages, methods of assay and primer design (6-8). Therefore, there is still an urgent need to explore more specific and sensitive biomarkers to help us better understand and manage MF. MicroRNAs (miRs) are short noncoding RNA molecules that play roles in posttranscriptional regulation by binding to RNA-induced silencing complexes and controlling physiological and pathological processes in various diseases (9). In addition, miRs play important roles in tumorigenesis and function as oncomiRs or tumor suppressors by regulating the levels of oncogenes or antioncogenes (10-12). The miR expression profiles in MF have been studied extensively and have shown a high correlation with disease progression, prognosis, and response to treatment (Table 1) (11-42). In this review, the function and molecular mechanism of miRs in the progression, diagnosis, variants, prognosis, and treatment of MF are discussed in detail (Figure 1).

\section{THE FUNCTION OF miR AND CLINICAL APPLICATION IN TUMOR}

miR is a short, noncoding RNA molecule that can regulate mRNA expression at the post-transcriptional level and is widely found in viruses, plants and animals. miR is broadly involved in a variety of physiological and pathological processes, and dysregulated $\mathrm{miR}$ expression is related to cancer initiation and progression (10). It does not encode functional proteins but can degrade or inhibit protein translation by means of complementary pairing with target mRNA and eventually inhibit specific gene expression (43).

In recent years, many studies have shown that miR is involved in the occurrence and development of tumors and has a broad clinical application space in the diagnosis and treatment of human cancers and hematological malignancies. Specifically,

TABLE 1 | miR expression profiles of available studies in MF.

\begin{tabular}{|c|c|c|c|}
\hline MiR ID & Expression & Functional role & Reference \\
\hline miR-155 & upregulate & diagnosis, progression, different variant (FMF), prognosis prediction, treatment & $(11,13-28)$ \\
\hline miR-203 & downregulate & diagnosis & $(16,19,24)$ \\
\hline miR-205 & downregulate & diagnosis(racial differences) & $(19,24)$ \\
\hline miR-92a & upregulate & progression, differential diagnosis, different variant(FMF, TMF) & $(15,23)$ \\
\hline miR-93-5p & upregulate & different variant(FMF,TMF) & $(13,29)$ \\
\hline \multirow[t]{2}{*}{ miR-93 } & upregulate & progression, differential Diagnosis, different variant(FMF), treatment & $(12,15,17,24,29-31)$ \\
\hline & downregulate & early diagnosis & $(32)$ \\
\hline miR-19b & upregulate & different variant(FMF) & (23) \\
\hline miR-34a & upregulate & different variant(FMF) & (13) \\
\hline miR-223 & upregulate & different variant(FMF), treatment response prediction & $(13,33)$ \\
\hline miR-191 & upregulate & treatment response prediction & (33) \\
\hline miR-342 & upregulate & treatment response prediction & (33) \\
\hline $\operatorname{miR}-181$ & upregulate & progression & (30) \\
\hline miR-181a & upregulate & diagnosis, progression, different variant(FMF,TMF) & $(13,30,34,35)$ \\
\hline miR-181b & upregulate & different variant(TMF) & (13) \\
\hline miR-338-3p & upregulate & prognosis prediction & $(36)$ \\
\hline $\operatorname{miR}-148 a-3 p$ & upregulate & prognosis prediction & (36) \\
\hline miR-106b & upregulate & progression, prognosis prediction & $(14,36,37)$ \\
\hline miR-106b-5p & upregulate & progression, prognosis prediction & (36) \\
\hline let-7a & downregulate & prognosis prediction & $(15)$ \\
\hline miR-17 92 & upregulate & progression, different variant(unilesional MF) & (38) \\
\hline $\mathrm{miR}-243$ & upregulate & diagnosis & $(16)$ \\
\hline miR-22 & downregulate & progression & (39) \\
\hline miR-200b & upregulate & diagnosis, good prognosis & $(16)$ \\
\hline miR-146a & upregulate & diagnosis & (34) \\
\hline miR-222 & not mentioned & diagnosis & (34) \\
\hline miR-26a & not mentioned & diagnosis & (34) \\
\hline miR-142-3p & upregulate & diagnosis & $(16)$ \\
\hline miR-130b & upregulate & differential diagnosis & $(16)$ \\
\hline miR-195-5p & downregulate & progression & $(37,40)$ \\
\hline miR-122 & upregulate & progression, treatment & (41) \\
\hline $\operatorname{miR}-15 a$ & downregulate & progression, prognosis prediction & (17) \\
\hline \multirow[t]{2}{*}{ miR-16 } & upregulate & progression, clinical course prediction & $(15,32)$ \\
\hline & downregulate & diagnosis, progression, clinical course prediction & $(17)$ \\
\hline miR-21 & upregulate & differentia diagnosis, clinical course prediction & $(14)$ \\
\hline
\end{tabular}

FMF, Folliculotropic mycosis fungoides; TMF, mycosis fungoides with larger cell transformation. 


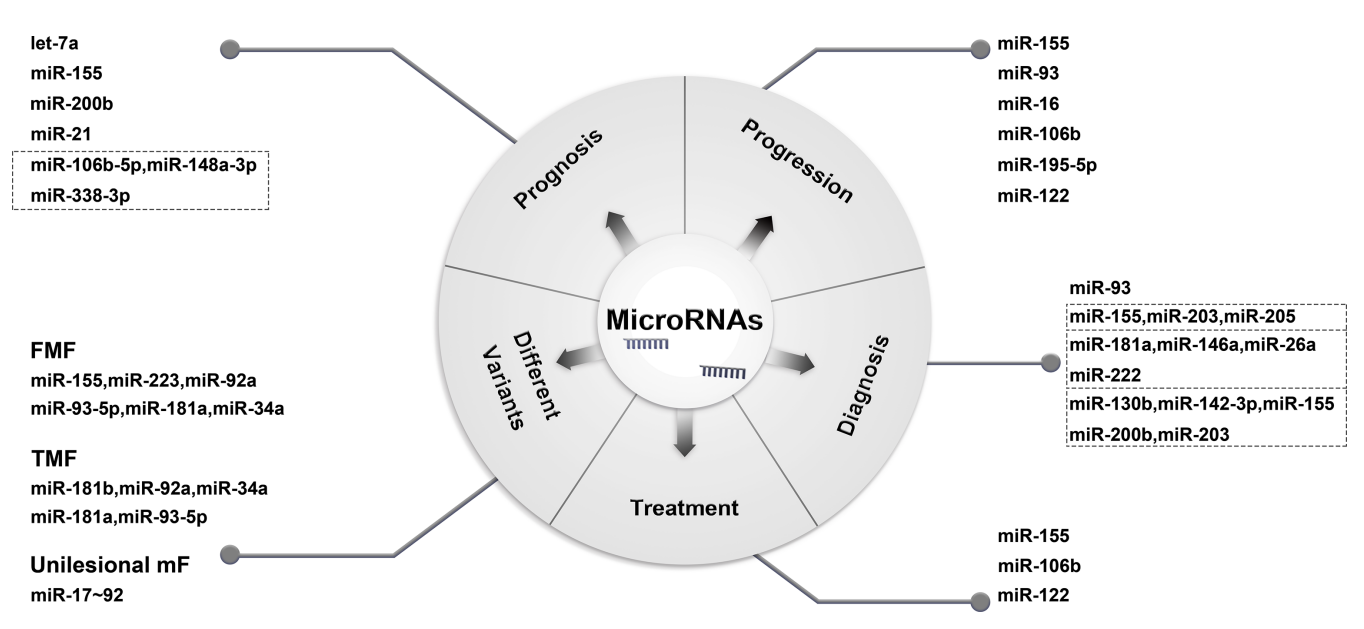

FIGURE 1 | Functions of miR in MF.

miR in serum or tumor tissue samples can be used as potential tumor markers for early diagnosis, such as breast cancer and Bcell lymphoma (44-46). In addition, studies have shown that miR can be used as a specific molecular target for targeted therapy $(9,47)$. Currently, miR mimics and miRNA inhibitors in the preclinical phase of drug development have shown potential as novel therapeutic drugs in tumor-treating fields.

\section{miR AND CUTANEOUS T-CELL LYMPHOMA (CTCL)}

CTCL comprises a heterogeneous group of disorders with variable clinical presentations, histological features, and prognoses. Growing evidence demonstrates that $\mathrm{miR}$ is involved in the development and progression of CTCL $(11,16$, $39,48-50)$. There are significant similarities and differences in the expression of miR among different variants of CTCL. For instance, miR-155, which was first identified as abnormally expressed in CTCLs, can be used as an oncogenic driver to promote tumor growth in both MF and anaplastic lymphoma kinase (ALK)-negative anaplastic large-cell lymphoma (ALCL) $(18,51)$. There exists a significant difference in the miR expression profile between tumoral MF, erythrodermic MF and the more aggressive leukemic variant of CTCL-Sezary syndrome (SS) - such as the miR-155, miR-21, miR-93, miR195-5p, and miR-17/92 (14, 15, 52, 53). Additional, Dercer expression have been served as an molecular marker in MF and might be of clinical relevance in MF, lymphomatoid papulosis and primary cutaneous CD4-positive small/medium T-cell lymphoma (54). To date, the mechanism of miR dysregulation in CTCL has not been fully elucidated and recognizing the abnormal expression of $\mathrm{miR}$ among different subgroups of CTCL variants, especially MF, is particularly important for elucidating the pathogenesis, early diagnosis, and identification of new therapeutic targets of CTCL.

\section{miR AND MF}

\section{miR Could Be a Gene Regulator in the Pathogenesis and Progression of MF}

The molecular pathogenesis of MF remains limited. miR may function as an oncogenic or tumor suppressor and contribute to the pathogenesis and progression of MF through interactions with specific target genes.

miR-155 acts as an oncogenic miR and is overexpressed in multiple solid tumors and B-cell lymphoma (55-57). Currently, it is one of the most intensively studied miRs in MF. Significant upregulation of miR-155 and miR-92a in tumoral MF was first observed by Van Kester et al. in 2011 (15). Subsequent studies further identified the overexpression of miR-155 in both early and advanced MF, and the expression level of miR-155 in biopsy samples increased with increasing clinical stage $(17,18,26)$. In vivo and in vitro experiments confirmed that miR-155 plays an important role in the development of $\mathrm{MF}$ and contributes to tumor growth by decreasing G2/M arrest and apoptosis (20). Additionally, previous studies indicated that the JAK/STAT5 pathway can promote the expression of miR-155 and promote the proliferation, growth, and survival of malignant MF cell lines in vitro (11). Interestingly, microbes have also been implicated in disease progression in CTCL $(58,59)$. A recent study showed that S. aureus and its enterotoxins might enhance miR-155 expression and promote disease progression by stimulating the expression of post-transcriptional regulators of malignant $\mathrm{T}$ cells (21).

Moreover, miR-93 has been described as an oncogene miR that can prevent apoptosis and promote tumor cell survival in various cancers $(12,31)$. miR-93 is overexpressed in advanced MF compared with inflammatory dermatosis and functions in the progression of $\operatorname{MF}(15,17,24,30)$. Gluud et al. found that miR-93 can interfere with the expression of tumor suppressor cyclin-dependent kinase inhibitor 1 (p21) in MF tumor T cell lines. In turn, the expression of the $\mathrm{p} 21$ protein was significantly increased in cells (MF2059 and MF3675) transfected with a miR- 
93-5p inhibitor, resulting in a $20-30 \%$ decrease in the proliferation of malignant $\mathrm{T}$ cell lines (29). In addition, Katona et al. observed a trend toward a loss of PTEN expression with histological progression of MF (60). The tumor suppressor gene PTEN is a known target of the miR-93-5p and miR-181 families $(61,62)$. However, contrary to the findings of previous studies, a recent study on miR-93 found that the expression of miR-93 was significantly downregulated in both early and advanced MF compared with normal and eczema cases (32). Therefore, further studies with larger cohorts of MF patients are needed to explore the role of miR-93 in the progression of MF.

In addition to miR-155 and miR-93, miR-16 dysregulation may also play a certain role in MF progression. Maj et al. found that the decrease in miR-15a and miR-16 is related to the development of advanced MF (17). This finding is consistent with the results of most previous studies regarding miR-16 as a tumor suppressor in various tumors, including pituitary adenomas and chronic lymphocytic leukemia (CLL) $(63,64)$. However, van Kester et al. observed that miR-16 in tumoral MF was upregulated compared with inflammatory dermatosis (15). Similarly, additional evidence suggests that miR-16 was significantly upregulated in advanced MF compared with patients at early stage and could be used to predict aggressive clinical course (32). Thus, more studies are needed to explore the specific expression and biological function of miR-16 in MF.

Recent studies have indicated that miRs not only play an important role in MF tumors but also in the tumor microenvironment (TME). miR-106b expression was observed in dermal T lymphocytes in skin lesions from patients with MF, and the expression level increased as the disease progressed. miR-106b can promote tumor proliferation in vitro by inhibiting the tumor suppressor p21 and thioredoxin-interacting protein (37). In addition, the local expression of miR-106b in stromal cells indicated that miR-106b may play potential roles in the MF TME. Microenvironment-mediated changes in miR expression in tumor cells mediating progression have also been highlighted. Research has shown that cancer-associated fibroblasts (CAFs) can protect MF cells from doxorubicin-induced cell death and promote migration through the secretion of CXCL12 (65). However, the role of miRs in MF progression between tumor cells and matrix components in the TME has not been elucidated. The value of the TME in exploring the pathogenesis of MF deserves further investigation.

In addition to the abovementioned oncogenic miRs, the cumulative inhibition of multiple tumor suppressor miRs may lead to the downregulation of multiple signaling pathways driving the disease progression of MF (66). miR195-5p may play a role as a tumor suppressor in MF, and its inhibitory effect is related to disease progression (37). The upregulation of miR195-5p inhibits cell cycle arrest through the downregulation of ADP-ribosylation factor-like protein 2 (ARL2), and low expression of miR-195-5p in MF skin lesions may promote disease progression (40). In addition, the role of miR-22 as a tumor suppressor in numerous solid tumors is widely accepted, and low miR-22 expression is associated with advanced stage and metastasis (67). In vitro studies have shown that miR-22 is significantly downregulated in malignant CTCL T cell lines (MyLa2059), and Jak3/STAT pathway-mediated inhibition of miR-22 may play a key role in CTCL pathogenesis and progression (39).

\section{miR Can Serve as Diagnostic Biomarkers in MF}

Early clinical and pathological diagnosis of MF remains a challenge because of its clinicopathological similarity to benign inflammatory disorders, which may also exhibit clonal TCR rearrangement in some conditions $(68,69)$. Also, the lack of specific molecular markers that can reliably differentiate the malignant $\mathrm{T}$-cells in MF from the reactive $\mathrm{T}$ cells in benign inflammatory disorders. Once entering the advanced stage, the median survival of patients with MF is only 1-5 years, with a 5year survival rate of less than $15 \%$ (70). Therefore, the search for diagnostic molecules is still needed for early diagnosis and then timely treatment.

With the deepening of research, there is growing evidence to support the biomarker potential of miRs for the diagnosis of cancer. miRs that have been confirmed to be related to the early diagnosis and differential diagnosis of MF include miR-93, miR146a, 146b-5p, miR-342-3p, miR-16, miR-181, miR-203, and miR-205 $(24,30,34,71)$. Specifically, miR-93 not only plays a role in the pathogenesis of MF but can also be used as a specific biomarker for the diagnosis of MF. The significant downregulation of miR-93 can be used for the early diagnosis of early challenging cases (32).

Although some single miRs do not have independent diagnostic value in MF, the specific combinations of miRs may achieve good diagnostic ability. As mentioned before, miR-155 plays an important role in the pathogenesis and progression of MF. However, given that higher miR-155 expression was also observed in T-cell-rich benign inflammatory dermatoses compared with early MF and folliculotropic MF (FMF), it cannot be used as a separate biomarker to distinguish early MF from benign inflammation dermatosis $(18,27)$. Interestingly, the combination of miR-155 with specific miRs has been proven to be of great value in differential diagnosis and early diagnosis in multiple studies. Ralfkiaer et al. developed a three-miR classifier composed of miR-155, miR-203, and miR-205 that can distinguish CTCL from benign inflammatory dermatosis with an accuracy of more than $90 \%$ (24). The strength of this classifier was also confirmed in subsequent independent cohort studies (71). Moreover, in view of the stability of miRs in serum or plasma, Dusilkova et al. established a plasma multiple miR classifier based on the upregulation of miR-155 and downregulation of $\mathrm{miR}-203 / \mathrm{miR}-205$ to detect CTCL with $100 \%$ specificity and $94 \%$ sensitivity, making routine clinical monitoring possible in the future (19). In addition, other investigations have suggested that a four-miR classifier composed of miR-181a, miR-146a, miR-222, and miR-26a could discriminate tumoral MF from benign inflammatory disease (34). Moreover, miR-181a and miR-146a may be used as specific biomarkers of MF and are significantly upregulated in both early and advanced $\operatorname{MF}(30,34,36)$. Importantly, there may 
be racial differences in the application scope of this classifier. An additional 5-miR diagnostic classifier, including miR-130b, miR142-3p, miR-155, miR-200b, miR-243, and miR-203, was established for the diagnosis and prognosis of CTCL in a study of an Asian population (16). Taken together, the diagnostic classifier combined with multiple miRs has better diagnostic intensity and accuracy and is expected to be a valuable adjunct in future clinical work.

\section{Is miR Differentially Expressed in Different Variants of MF?}

According to its clinicopathologic characteristics, MF can be divided into several variants (72). Folliculotropic MF (FMF) is a rare variant of MF with distinct clinicopathological features. The clinical course and treatment response varies according to different stage of disease (early- and advanced-stage) $(73,74)$. Specifically, patients with FMF presenting with only patches and/ or follicular papules (early-stage) had a favorable prognosis with a 5-year overall survival (OS) of 92\%, while patients with FMF presenting with tumors and/or nodules (advanced-stage) had a 5 -year OS of 50\%. Additionally, a small number of MF patients may undergo large cell transformation (TMF), which is characterized by an aggressive clinical course and refraction to systemic therapies including anthracyclines, bexarotene, methotrexate with a median survival of 18.4 to 24 months (7577). However, the molecular background of FMF and TMF has not been fully elucidated. Marosvári $\mathrm{D}$ et al. showed for the first time in 2015 that miR-93-5p, miR-181a, and miR-34a were significantly upregulated in FMF and TMF. Overexpression of miR-155 and miR-223 was also observed in FMF (13). Additionally, Garaicoa et al. compared the miR expression profile among tumoral MF, FMF, and TMF and found that the expression levels of miR-19b, miR-92a, and miR-155 in FMF and TMF were higher than those in tumoral MF (23). In recent years, some scholars have proposed that according to different clinicopathological features, FMF can be categorized in early stage with indolent clinical course and advanced/tumoral stage requiring aggressive treatment $(74,78)$. Atzmony et al. found that there was a significant difference in miR-155 expression between early and tumoral FMF, but there was no significant difference in miR-155 expression between early FMF and MF or between tumoral FMF and MF (27). To some extent, this finding confirms that there might be two different stages of FMF, but the relationship between MF and FMF in different stages still needs further research and exploration to provide a theoretical basis for personalized treatment of different stages and subtypes of MF. In addition, the study showed that miR-181b and miR-93-5p were highly expressed in the TMF, while the level of miR-155 was not significantly increased, indicating that $\mathrm{miR}-181 \mathrm{~b}$ and $\mathrm{miR}-93-5 \mathrm{p}$ may play a role in the pathogenesis of the TMF, while the regulation of miR-155-related gene expression may not be involved in large cell transformation (13).

In some special cases, it is difficult to distinguish erythroderma MF (eMF) from Sézary Syndrome (SS) clinically and histologically. Due to the differences in treatment recommendations and prognosis, it is necessary to distinguish between eMF and SS. Rittig et al. found that there was a significant difference in the $\mathrm{miR}$ expression profile between eMF and SS. In particular, the expression levels of miR-106b, miR-155, and miR-21 in eMF were significantly lower than those in SS (14).

Unilesional MF is characterized by a solitary erythematous patch or plaque located on the trunk and upper extremities clinically and is histologically indistinguishable from typical MF. Unlike early MF, unilesional MF can maintain a benign clinical course without any treatment, and there is no obvious recurrence after treatment. Studies have shown that the miR expression profile of unilesional MF is different from that of early MF. The former has a high level of miR-17 92 members and is accompanied by Th1 skewing (38). The antitumor activity of miR-17 92 has been confirmed in numerous previous studies $(79,80)$. These findings suggest that there is a strong reactive $\mathrm{T}$ cell immune response in unilesional MF, which may explain the locality of the disease.

\section{Existing miR-Based Therapeutics and Potential Treatment Options in MF}

Local and systemic therapies available for MF have reduced tumor burden and improved quality of life. However, classic regimens based on anthracycline or nucleoside analogs can only obtain a short-lived response and have had limited impact on the survival of patients with advanced $\operatorname{MF}(81,82)$. Therefore, advances in MF treatment research are focused on identifying new pharmacological targets. Current clinical trials show that miR-based treatment seems to be feasible (83). Given the important role of miR-155 in MF, Moyal et al. found that miR-155 promotes tumor growth in xenografted MF mice by reducing apoptosis. Anti-miR-155 can be used as monotherapy or in combination with apoptosis therapy and cell cycle checkpoint inhibitors to improve the effectiveness of MF therapy (20). Indeed, clinical trials were conducted to test the therapeutic efficacy of a miR-155-targeting nucleic acid modification inhibitor called cobomarsen (MRG-106) in MF in 2018 (26). Cobomarsen can reduce the expression of multiple gene pathways related to cell survival by blocking miR-155 and finally reduce cell proliferation and activate apoptosis. Therefore, it can be suggested that cobomarsen can potentially be used as a therapeutic agent for MF (26). Surprisingly, active antimicrobial therapy can inhibit the activity of MF by affecting the expression of miR-155 $(22,25)$. Indeed, Duvich et al. reported that the combination of antibiotics, a germicidal whirlpool bath system, and steroids has a significant effect on patients with SS. In vitro experiments demonstrated that active antibiotic therapy may inhibit malignant $\mathrm{T}$ cell proliferation in advanced CTCL by inhibiting the staphylococcal enterotoxin (SE)-mediated bystander $\mathrm{T}$ cell response (35). Recent studies have further shown that this inhibitory effect may be achieved by partially reversing SE-induced pathological processes involving STAT5 and miR-155 (21). Notably, Staphylococcus aureus is also present to a greater extent in skin lesions in patients with MF than in patients with non-lesional or healthy skin. miR-155 may be associated with secondary skin infection in patients with MF. 
Additionally, the involvement of other infectious agents in MF, such as Borrelia burgdorferi, Chlamydophila, have been described (84). A previous study by Tothova et al. found that Borrelia might exert its causative role in MF through a chronic type-1 immune response with interferon- $\gamma$ production (85). Besides, evidence of type C pneumonia in MF biopsies suggests an association between Chlamydophila infection and MF development (86). However, further studies are needed to assess the efficacy of anti-Borrelia therapy in treating Borreliapositive MF and verify the correlation between Chlamydophila and MF in larger cohorts. In view of the important role of antimicrobial therapy and miRs in MF, the combination of miRbased therapy and antimicrobial therapy may produce synergistic therapeutic effects and improve the therapeutic efficacy of patients with MF, especially for advanced MF with large-area involvement.

Interestingly, miR-106b was previously known to provide the strongest prognostic prediction of a high risk of progression (87). Given that miR-106b is also highly expressed in the early stage of MF, the development of miR-106b inhibitors applied in the early stage of the disease may prevent or delay disease progression (36). In addition, inhibition of miR-93 resulted in decreased proliferation of malignant $\mathrm{T}$ cell lines. The effect of this reduction in proliferation was similar to that observed following inhibition of miR-155 (11, 29, 88). Thus, anti-miR-93 could be a valuable therapeutic agent for patients with MF. Furthermore, researchers found that miR-122 was overexpressed in advanced MF, reducing sensitivity to chemotherapy-induced apoptosis through signal transduction circuits involved in Akt activation and p53 inhibition. This finding provides a new idea for the use of chemotherapy sensitizers in advanced MF (41). In addition to the development of the abovementioned miR-based therapeutic drugs, the miR expression spectrum can also predict treatment response. Studies have shown that MF patients with rapidly elevated levels of miR-223, miR-191, and miR-342 after extracorporeal blood collection are more likely to show good clinical responses after 6-12 months of treatment (42).

\section{miR May Play an Important Role in Predicting Prognosis in MF}

miRs not only contribute to increasing the accuracy of diagnosis but can also be used to predict prognosis. Several studies have emphasized that miRs can be used as a potential prognostic biomarker in MF. For instance, the miRs of the let-7 family are downregulated in advanced and metastatic MF. The prognosis of patients with low expression of let-7a was worse than that of other patients (15). Of note, although multiple studies have shown that miR-155 can be used as a prognostic indicator of CTCL, its prognostic value in MF and the relationship between the intensity of miR-155 expression and disease stage are discrepant $(16,17,26)$. Shen et al. found that miR-155 was associated with worse clinical outcomes in an Asian population with a total of 158 CTCLs (including MF, cutaneous anaplastic large cell lymphoma, peripheral T cell lymphoma and NK/T cell lymphoma). However, excluding other types of CTCL than MF, the expression of miR-
155 had no significant correlation with the overall survival of patients with different stages of MF (16). The inconsistency in the above findings may be attributed to the different ethnic backgrounds of the patients and varied numbers of patients with different disease stages enrolled in the above studies. Comparatively, miR-200b is significantly associated with the overall survival of MF patients regardless of disease stage (16). High miR-200b expression implied favorable prognosis. Taken together, there may be differences in prognostic markers among subgroups of CTCLs, and it is necessary to develop specific prognostic biomarkers for MF patients.

Early identification of patients at a higher risk of progression may facilitate more individualized treatment of these patients. Therefore, it is worth drawing attention to the prognostic stratification of patients with early MF. Lindahl et al. developed and verified a 3-miR prognostic classifier based on miR-106b-5p, miR-148a-3p, and miR-338-3p, which can successfully divide patients into high- and low-risk groups for disease progression. This 3-miR classifier may be an effective tool to predict the progression of early MF at the time of diagnosis and has important prognostic value (36). Among them, miR$106 \mathrm{~b}$ is the most powerful prognostic marker of disease progression in MF. As the disease progresses, miR-106b can regulate the expression of the tumor suppressor genes p21 and thioredoxin-interacting protein (TXNIP) and promote the proliferation of tumor cells in MF (37). Additionally, there were also differences in the expression levels of miR-16, miR93, and miR-106a between progressive and nonprogressive patients (30). Of note, there may also be racial differences in the application scope of this classifier. Shen et al. verified that miR-155 and miR-200b can be used as effective predictors of clinical outcome in Asian populations (16). Therefore, it is necessary to develop and validate prognostic miR classifiers among populations with different racial backgrounds.

\section{Future Perspectives of Exosomal miR-Based Therapeutics in MF}

In addition to the tumor cells themselves, the malignant tumor phenotype can also transmit genetic information, including miRs, to other cells in the tumor microenvironment through exosomes, which can promote proliferation, angiogenesis, metastasis, and drug resistance. Moyal et al. first identified miR-155 and miR-1246 in exosomes derived from MF cell lines in 2021. It was confirmed that exomiR-155 can promote the motility of MF cell lines in vitro. In addition, exomiR-1246 expression levels appeared to be correlated with MF staging, and were significantly higher in plaque/tumor MF patients than in healthy population (28). Moreover, exosomal miRs can affect extracellular matrix (ECM) and immune system activation and recruitment to promote tumor cell survival. Furthermore, exosomal miRs can be used as promising noninvasive biomarkers and potential targeting factors and delivery vehicles for tumor diagnosis and treatment. Therefore, exosomal miRbased therapeutics are a potential feasible candidate therapy for MF. 


\section{CONCLUSION}

MF is the most common cutaneous T-cell lymphoma. The main problem in the diagnosis and treatment of MF is the inefficient methods of early diagnosis and short-lived response to classical chemotherapy. The emergence of the role of miR in cancer progression has prompted us to elucidate the prospects of $\mathrm{miR}$ as new therapeutic target. Several studies have identified them as potential diagnostic and prognostic biomarkers in MF. The identification of these new gene regulatory targets opens up a new field for the diagnosis, treatment and prognosis of MF. However, aberrant miR expression have been detected in a variety inflammatory disease and malignancies, and diseasespecific miR remains as one of the most challenging issues. Additionally, the functions of partial miRs in MF remain

\section{REFERENCES}

1. Saunes M, Nilsen TI, Johannesen TB. Incidence of Primary Cutaneous T-Cell Lymphoma in Norway. Br J Dermatol (2009) 160(2):376-9. doi: 10.1111/ j.1365-2133.2008.08852.x

2. Hristov AC, Tejasvi T, Wilcox RA. Mycosis Fungoides and Sezary Syndrome: 2019 Update on Diagnosis, Risk-Stratification, and Management. Am J Hematol (2019) 94(9):1027-41. doi: 10.1002/ajh.25577

3. Agar NS, Wedgeworth E, Crichton S, Mitchell TJ, Cox M, Ferreira S, et al. Survival Outcomes and Prognostic Factors in Mycosis Fungoides/Sezary Syndrome: Validation of the Revised International Society for Cutaneous Lymphomas/European Organisation for Research and Treatment of Cancer Staging Proposal. J Clin Oncol (2010) 28(31):4730-9. doi: 10.1200/ JCO.2009.27.7665

4. Wong HK, Mishra A, Hake T, Porcu P. Evolving Insights in the Pathogenesis and Therapy of Cutaneous T-Cell Lymphoma (Mycosis Fungoides and Sezary Syndrome). Br J Haematol (2011) 155(2):150-66. doi: 10.1111/j.13652141.2011.08852.x

5. Thurber SE, Zhang B, Kim YH, Schrijver I, Zehnder J, Kohler S. T-Cell Clonality Analysis in Biopsy Specimens From Two Different Skin Sites Shows High Specificity in the Diagnosis of Patients With Suggested Mycosis Fungoides. J Am Acad Dermatol (2007) 57(5):782-90.

6. Hodges E, Krishna MT, Pickard C, Smith JL. Diagnostic Role of Tests for T Cell Receptor (TCR) Genes. J Clin Pathol (2003) 56(1):1-11. doi: 10.1136/ jcp.56.1.1

7. Ponti R, Fierro MT, Quaglino P, Lisa B, Paola F, Michela O, et al. TCRgamma-Chain Gene Rearrangement by PCR-Based GeneScan: Diagnostic Accuracy Improvement and Clonal Heterogeneity Analysis in Multiple Cutaneous T-Cell Lymphoma Samples. J Invest Dermatol (2008) 128 (4):1030-8. doi: 10.1038/sj.jid.5701109

8. Phyo ZH, Shanbhag S, Rozati S. Update on Biology of Cutaneous T-Cell Lymphoma. Front Oncol (2020) 10:765. doi: 10.3389/fonc.2020.00765

9. Rupaimoole R, Slack FJ. MicroRNA Therapeutics: Towards a New Era for the Management of Cancer and Other Diseases. Nat Rev Drug Discovery (2017) 16(3):203-22. doi: 10.1038/nrd.2016.246

10. Calin GA, Croce CM. MicroRNA Signatures in Human Cancers. Nat Rev Cancer (2006) 6(11):857-66. doi: 10.1038/nrc1997

11. Kopp KL, Ralfkiaer U, Gjerdrum LM, Helvad R, Pedersen IH, Litman T, et al. STAT5-Mediated Expression of Oncogenic miR-155 in Cutaneous T-Cell Lymphoma. Cell Cycle (2013) 12(12):1939-47. doi: 10.4161/cc.24987

12. Li N, Miao Y, Shan Y, Liu B, Li Y, Zhao L, et al. MiR-106b and miR-93 Regulate Cell Progression by Suppression of PTEN via PI3K/Akt Pathway in Breast Cancer. Cell Death Dis (2017) 8(5):e2796. doi: 10.1038/cddis.2017.119

13. Marosvari D, Teglasi V, Csala I, Marschalko M, Bodor C, Timar B, et al. Altered microRNA Expression in Folliculotropic and Transformed Mycosis Fungoides. Pathol Oncol Res (2015) 21(3):821-5. doi: 10.1007/s12253-0159897-8 controversial and further investigation and validation in larger cohorts are needed. Also, miR-based therapeutics in CTCL is still in budding stages, the reliable and targeted site-specific delivery of miR might be a major obstacle to the use of miR-based therapy. Thus, it is urgently needed to overcome the above obstacles before development of novel miR-based therapeutics strategy.

\section{AUTHOR CONTRIBUTIONS}

PFW designed and wrote this article. YX made a contribution to revision. LW designed the whole project and revised the final manuscript. All authors contributed to the article and approved the submitted version.

14. Rittig AH, Lindahl LM, Johansen C, Celis P, Odum N, Iversen L, et al. The MicroRNA Expression Profile Differs Between Erythrodermic Mycosis Fungoides and Sezary Syndrome. Acta Derm Venereol (2019) 99(12):114853. doi: 10.2340/00015555-3306

15. van Kester MS, Ballabio E, Benner MF, Chen XH, Saunders NJ, van der Fits L, et al. miRNA Expression Profiling of Mycosis Fungoides. Mol Oncol (2011) 5 (3):273-80. doi: 10.1016/j.molonc.2011.02.003

16. Shen X, Wang B, Li K, Wang L, Zhao X, Xue F, et al. MicroRNA Signatures in Diagnosis and Prognosis of Cutaneous T-Cell Lymphoma. J Invest Dermatol (2018) 138(9):2024-32. doi: 10.1016/j.jid.2018.03.1500

17. Maj J, Jankowska-Konsur A, Sadakierska-Chudy A, Noga L, Reich A. Altered microRNA Expression in Mycosis Fungoides. Br J Dermatol (2012) 166 (2):331-6. doi: 10.1111/j.1365-2133.2011.10669.x

18. Moyal L, Barzilai A, Gorovitz B, Hirshberg A, Amariglio N, Jacob-Hirsch J, et al. miR-155 Is Involved in Tumor Progression of Mycosis Fungoides. Exp Dermatol (2013) 22(6):431-3. doi: 10.1111/exd.12161

19. Dusilkova N, Basova P, Polivka J, Kodet O, Kulvait V, Pesta M, et al. Plasma miR-155, miR-203, and miR-205 Are Biomarkers for Monitoring of Primary Cutaneous T-Cell Lymphomas. Int J Mol Sci (2017) 18(10). doi: 10.3390/ ijms 18102136

20. Moyal L, Yehezkel S, Gorovitz B, Keren A, Gilhar A, Lubin I, et al. Oncogenic Role of microRNA-155 in Mycosis Fungoides: An In Vitro and Xenograft Mouse Model Study. Br J Dermatol (2017) 177(3):791-800. doi: 10.1111/ bjd. 15422

21. Willerslev-Olsen A, Gjerdrum LMR, Lindahl LM, Buus TB, Pallesen EMH, Gluud M, et al. Staphylococcus Aureus Induces Signal Transducer and Activator of Transcription 5dependent miR-155 Expression in Cutaneous T-Cell Lymphoma. J Invest Dermatol (2021). doi: 10.1016/j.jid.2021.01.038

22. Lewis DJ, Holder BB, Duvic M. The "Duvic Regimen" for Erythrodermic Flares Secondary to Staphylococcus Aureus in Mycosis Fungoides and Sezary Syndrome. Int J Dermatol (2018) 57(1):123-4. doi: 10.1111/ijd.13832

23. Garaicoa FH, Roisman A, Arias M, Trila C, Fridmanis M, Abeldano A, et al. Genomic Imbalances and microRNA Transcriptional Profiles in Patients With Mycosis Fungoides. Tumour Biol (2016) 37(10):13637-47. doi: 10.1007/s13277-016-5259-8

24. Ralfkiaer U, Hagedorn PH, Bangsgaard N, Lovendorf MB, Ahler CB, Svensson L, et al. Diagnostic microRNA Profiling in Cutaneous T-Cell Lymphoma (CTCL). Blood (2011) 118(22):5891-900. doi: 10.1182/blood-2011-06-358382

25. Talpur R, Bassett R, Duvic M. Prevalence and Treatment of Staphylococcus Aureus Colonization in Patients With Mycosis Fungoides and Sezary Syndrome. Br J Dermatol (2008) 159(1):105-12. doi: 10.1111/j.13652133.2008.08612.x

26. Seto AG, Beatty X, Lynch JM, Hermreck M, Tetzlaff M, Duvic M, et al. Cobomarsen, an Oligonucleotide Inhibitor of miR-155, Co-Ordinately Regulates Multiple Survival Pathways to Reduce Cellular Proliferation and Survival in Cutaneous T-Cell Lymphoma. Br J Haematol (2018) 183(3):42844. doi: 10.1111/bjh.15547 
27. Atzmony L, Moyal L, Feinmesser M, Gorovitz B, Hirshberg A, Amitay-Laish I, et al. Stage-Dependent Increase in Expression of miR-155 and Ki-67 and Number of Tumour-Associated Inflammatory Cells in Folliculotropic Mycosis Fungoides. Acta Derm Venereol (2020) 100(15):adv00230. doi: 10.2340/00015555-3578

28. Moyal L, Arkin C, Gorovitz-Haris B, Querfeld C, Rosen S, Knaneh J, et al. Mycosis Fungoides-Derived Exosomes Promote Cell Motility and Are Enriched With microRNA-155 and microRNA-1246, and Their PlasmaCell-Free Expression May Serve as a Potential Biomarker for Disease Burden. Br J Dermatol (2021). doi: 10.1111/bjd.20519

29. Gluud M, Fredholm S, Blumel E, Willerslev-Olsen A, Buus TB, Nastasi C, et al. MicroRNA-93 Targets P21 and Promotes Proliferation in Mycosis Fungoides T Cells. Dermatology (2021) 237(2):277-82. doi: 10.1159/ 000505743

30. Ralfkiaer U, Lindahl LM, Litman T, Gjerdrum LM, Ahler CB, Gniadecki R, et al. MicroRNA Expression in Early Mycosis Fungoides Is Distinctly Different From Atopic Dermatitis and Advanced Cutaneous T-Cell Lymphoma. Anticancer Res (2014) 34(12):7207-17.

31. Hanahan D, Weinberg RA. The Hallmarks of Cancer. Cell (2000) 100(1):5770. doi: 10.1016/s0092-8674(00)81683-9

32. Talaat IM, Abdelmaksoud RE, Guimei M, Agamia NF, Nugud A, El-Serafi AT. Potential Role for microRNA-16 (miR-16) and microRNA-93 (miR-93) in Diagnosis and Prediction of Disease Progression in Mycosis Fungoides in Egyptian Patients. PloS One (2019) 14(10):e0224305. doi: 10.1371/ journal.pone.0224305

33. McGirt LY, Adams CM, Baerenwald DA, Zwerner JP, Zic JA, Eischen CM. miR-223 Regulates Cell Growth and Targets Proto-Oncogenes in Mycosis Fungoides/Cutaneous T-Cell Lymphoma. J Invest Dermatol (2014) 134 (4):1101-7. doi: 10.1038/jid.2013.461

34. Manso R, Martinez-Magunacelaya N, Erana-Tomas I, Monsalvez V, Rodriguez-Peralto JL, Ortiz-Romero PL, et al. Mycosis Fungoides Progression Could be Regulated by microRNAs. PloS One (2018) 13(6): e0198477. doi: 10.1371/journal.pone.0198477

35. Lindahl LM, Willerslev-Olsen A, Gjerdrum LMR, Nielsen PR, Blumel E, Rittig $\mathrm{AH}$, et al. Antibiotics Inhibit Tumor and Disease Activity in Cutaneous T-Cell Lymphoma. Blood (2019) 134(13):1072-83. doi: 10.1182/blood.2018888107

36. Lindahl LM, Besenbacher S, Rittig AH, Celis P, Willerslev-Olsen A, Gjerdrum LMR, et al. Prognostic miRNA Classifier in Early-Stage Mycosis Fungoides: Development and Validation in a Danish Nationwide Study. Blood (2018) 131 (7):759-70. doi: 10.1182/blood-2017-06-788950

37. Lindahl LM, Gluud M, Emmanuel T, Thomsen EA, Hu T, Rittig AH, et al. MicroRNA-106b Regulates Expression of the Tumour Suppressors P21 and TXNIP and Promotes Tumour Cell Proliferation in Mycosis Fungoides. Acta Derm Venereol (2020) 100(16):adv00270. doi: 10.2340/00015555-3574

38. Moyal L, Gorovitz-Haris B, Yehezkel S, Jacob-Hirsch J, Bershtein V, Barzilai A, et al. Unilesional Mycosis Fungoides Is Associated With Increased Expression of microRNA-17 92 and T Helper 1 Skewing. Br J Dermatol (2019) 180(5):1123-34. doi: 10.1111/bjd.17425

39. Sibbesen NA, Kopp KL, Litvinov IV, Jonson L, Willerslev-Olsen A, Fredholm S, et al. Jak3, STAT3, and STAT5 Inhibit Expression of miR-22, a Novel Tumor Suppressor microRNA, in Cutaneous T-Cell Lymphoma. Oncotarget (2015) 6(24):20555-69. doi: 10.18632/oncotarget.4111

40. Rittig AH, Johansen C, Celis P, Odum N, Litman T, Woetmann A, et al. Suppressed microRNA-195-5p Expression in Mycosis Fungoides Promotes Tumor Cell Proliferation. Exp Dermatol (2020). doi: 10.1111/exd.14124

41. Manfe V, Biskup E, Rosbjerg A, Kamstrup M, Skov AG, Lerche CM, et al. miR-122 Regulates P53/Akt Signalling and the Chemotherapy-Induced Apoptosis in Cutaneous T-Cell Lymphoma. PloS One (2012) 7(1):e29541. doi: 10.1371/journal.pone.0029541

42. McGirt LY, Baerenwald DA, Vonderheid EC, Eischen CM. Early Changes in miRNA Expression Are Predictive of Response to Extracorporeal Photopheresis in Cutaneous T-Cell Lymphoma. J Eur Acad Dermatol Venereol (2015) 29(11):2269-71. doi: 10.1111/jdv.12571

43. Morris KV, Chan SW, Jacobsen SE, Looney DJ. Small Interfering RNAInduced Transcriptional Gene Silencing in Human Cells. Science (2004) 305 (5688):1289-92. doi: 10.1126/science.1101372

44. Duffy J, Padovani F, Brunetti G, Noy P, Certa U, Hegner M. Towards Personalised Rapid Label Free miRNA Detection for Cancer and Liver
Injury Diagnostics in Cell Lysates and Blood Based Samples. Nanoscale (2018) 10(26):12797-804. doi: 10.1039/c8nr03604g

45. Jørgensen S, Paulsen IW, Hansen JW, Tholstrup D, Hother C, Sørensen E, et al. The Value of Circulating microRNAs for Early Diagnosis of B-Cell Lymphoma: A Case-Control Study on Historical Samples. Sci Rep (2020) 10 (1):9637. doi: 10.1038/s41598-020-66062-1

46. Wang Y, Yin W, Lin Y, Yin K, Zhou L, Du Y, et al. Downregulated Circulating microRNAs After Surgery: Potential Noninvasive Biomarkers for Diagnosis and Prognosis of Early Breast Cancer. Cell Death Discovery (2018) 4:21. doi: 10.1038/s41420-018-0089-7

47. Li Z, Rana TM. Therapeutic Targeting of microRNAs: Current Status and Future Challenges. Nat Rev Drug Discovery (2014) 13(8):622-38. doi: 10.1038/ $\operatorname{nrd} 4359$

48. Papadavid E, Braoudaki M, Bourdakou M, Lykoudi A, Nikolaou V, Tounta G, et al. Aberrant microRNA Expression in Tumor Mycosis Fungoides. Tumour Biol (2016) 37(11):14667-75. doi: 10.1007/s13277-016-5325-2

49. Gluud M, Willerslev-Olsen A, Gjerdrum LMR, Lindahl LM, Buus TB, Andersen MH, et al. MicroRNAs in the Pathogenesis, Diagnosis, Prognosis and Targeted Treatment of Cutaneous T-Cell Lymphomas. Cancers (Basel) (2020) 12(5). doi: 10.3390/cancers 12051229

50. Sandoval J, Diaz-Lagares A, Salgado R, Servitje O, Climent F, Ortiz-Romero PL, et al. MicroRNA Expression Profiling and DNA Methylation Signature for Deregulated microRNA in Cutaneous T-Cell Lymphoma. J Invest Dermatol (2015) 135(4):1128-37. doi: 10.1038/jid.2014.487

51. Merkel O, Hamacher F, Griessl R, Grabner L, Schiefer AI, Prutsch N, et al. Oncogenic Role of miR-155 in Anaplastic Large Cell Lymphoma Lacking the T(2;5) Translocation. J Pathol (2015) 236(4):445-56. doi: 10.1002/path.4539

52. Narducci MG, Arcelli D, Picchio MC, Lazzeri C, Pagani E, Sampogna F, et al. MicroRNA Profiling Reveals That miR-21, Mir486 and miR-214 Are Upregulated and Involved in Cell Survival in Sezary Syndrome. Cell Death Dis (2011) 2:e151. doi: $10.1038 /$ cddis. 2011.32

53. Ballabio E, Mitchell T, van Kester MS, Taylor S, Dunlop HM, Chi J, et al. MicroRNA Expression in Sezary Syndrome: Identification, Function, and Diagnostic Potential. Blood (2010) 116(7):1105-13. doi: 10.1182/blood-200912-256719

54. Valencak J, Schmid K, Trautinger F, Wallnöfer W, Muellauer L, Soleiman A, et al. High Expression of Dicer Reveals a Negative Prognostic Influence in Certain Subtypes of Primary Cutaneous T Cell Lymphomas. J Dermatol science (2011) 64(3):185-90. doi: 10.1016/j.jdermsci.2011.08.011

55. Ahmadvand M, Eskandari M, Pashaiefar H, Yaghmaie M, Manoochehrabadi S, Khakpour G, et al. Over Expression of Circulating miR-155 Predicts Prognosis in Diffuse Large B-Cell Lymphoma. Leuk Res (2018) 70:45-8. doi: 10.1016/j.leukres.2018.05.006

56. Patel GK, Khan MA, Bhardwaj A, Srivastava SK, Zubair H, Patton MC, et al. Exosomes Confer Chemoresistance to Pancreatic Cancer Cells by Promoting ROS Detoxification and miR-155-Mediated Suppression of Key GemcitabineMetabolising Enzyme, DCK. Br J Cancer (2017) 116(5):609-19. doi: 10.1038/ bjc. 2017.18

57. Volinia S, Calin GA, Liu CG, Ambs S, Cimmino A, Petrocca F, et al. A microRNA Expression Signature of Human Solid Tumors Defines Cancer Gene Targets. Proc Natl Acad Sci USA (2006) 103(7):2257-61. doi: 10.1073/pnas.0510565103

58. Mirvish ED, Pomerantz RG, Geskin LJ. Infectious Agents in Cutaneous T-Cell Lymphoma. J Am Acad Dermatol (2011) 64(2):423-31. doi: 10.1016/ j.jaad.2009.11.692

59. Mirvish JJ, Pomerantz RG, Falo LDJr., Geskin LJ. Role of Infectious Agents in Cutaneous T-Cell Lymphoma: Facts and Controversies. Clin Dermatol (2013) 31(4):423-31. doi: 10.1016/j.clindermatol.2013.01.009

60. Katona TM, Smoller BR, Webb AL, Hattab EM, Khalil A, Hiatt KM. Expression of PTEN in Mycosis Fungoides and Correlation With Loss of Heterozygosity. Am J Dermatopathol (2013) 35(5):555-60. doi: 10.1097/ DAD.0b013e318276cc68

61. Hsu SD, Tseng YT, Shrestha S, Lin YL, Khaleel A, Chou CH, et al. Mirtarbase Update 2014: An Information Resource for Experimentally Validated miRNA-Target Interactions. Nucleic Acids Res (2014) 42(Database issue): D78-85. doi: 10.1093/nar/gkt1266

62. Williams A, Henao-Mejia J, Harman CC, Flavell RA. miR-181 and Metabolic Regulation in the Immune System. Cold Spring Harb Symp Quant Biol (2013) 78:223-30. doi: 10.1101/sqb.2013.78.020024 
63. Nicoloso MS, Kipps TJ, Croce CM, Calin GA. MicroRNAs in the Pathogeny of Chronic Lymphocytic Leukaemia. Br J Haematol (2007) 139(5):709-16. doi: 10.1111/j.1365-2141.2007.06868.x

64. Bottoni A, Piccin D, Tagliati F, Luchin A, Zatelli MC, degli Uberti EC. miR15a and miR-16-1 Down-Regulation in Pituitary Adenomas. J Cell Physiol (2005) 204(1):280-5. doi: 10.1002/jcp.20282

65. Aronovich A, Moyal L, Gorovitz B, Amitay-Laish I, Naveh HP, Forer Y, et al. Cancer-Associated Fibroblasts in Mycosis Fungoides Promote Tumor Cell Migration and Drug Resistance Through CXCL12/CXCR4. J Invest Dermatol (2021) 141(3):619-627 e2. doi: 10.1016/j.jid.2020.06.034

66. Odum N. Deregulated Signalling and Inflammation in Cutaneous T-Cell Lymphoma. Br J Dermatol (2020) 182(1):16-7. doi: 10.1111/bjd.18353

67. Li B, Song Y, Liu TJ, Cui YB, Jiang Y, Xie ZS, et al. miRNA-22 Suppresses Colon Cancer Cell Migration and Invasion by Inhibiting the Expression of TCell Lymphoma Invasion and Metastasis 1 and Matrix Metalloproteinases 2 and 9. Oncol Rep (2013) 29(5):1932-8. doi: 10.3892/or.2013.2300

68. Pimpinelli N, Olsen EA, Santucci M, Vonderheid E, Haeffner AC, Stevens S, et al. Defining Early Mycosis Fungoides. J Am Acad Dermatol (2005) 53 (6):1053-63. doi: 10.1016/j.jaad.2005.08.057

69. Keehn CA, Belongie IP, Shistik G, Fenske NA, Glass LF. The Diagnosis, Staging, and Treatment Options for Mycosis Fungoides. Cancer Control (2007) 14(2):102-11. doi: 10.1177/107327480701400203

70. Wilcox RA. Cutaneous T-Cell Lymphoma: 2016 Update on Diagnosis, RiskStratification, and Management. Am J Hematol (2016) 91(1):151-65. doi: 10.1002/ajh.24233

71. Marstrand T, Ahler CB, Ralfkiaer U, Clemmensen A, Kopp KL, Sibbesen NA, et al. Validation of a Diagnostic microRNA Classifier in Cutaneous T-Cell Lymphomas. Leuk Lymphoma (2014) 55(4):957-8. doi: 10.3109/ 10428194.2013.815352

72. Swerdlow SH, Campo E, Pileri SA, Harris NL, Stein H, Siebert R, et al. The 2016 Revision of the World Health Organization Classification of Lymphoid Neoplasms. Blood (2016) 127(20):2375-90. doi: 10.1182/blood-2016-01643569

73. van Santen S, van Doorn R, Neelis KJ, Daniëls LA, Horváth B, Bruijn MS, et al. Recommendations for Treatment in Folliculotropic Mycosis Fungoides: Report of the Dutch Cutaneous Lymphoma Group. Br J Dermatol (2017) 177(1):223-8. doi: 10.1111/bjd.15355

74. van Santen S, Roach RE, van Doorn R, Horvath B, Bruijn MS, Sanders CJ, et al. Clinical Staging and Prognostic Factors in Folliculotropic Mycosis Fungoides. JAMA Dermatol (2016) 152(9):992-1000. doi: 10.1001/jamadermatol. 2016.1597

75. Vergier B, de Muret A, Beylot-Barry M, Vaillant L, Ekouevi D, Chene G, et al. Transformation of Mycosis Fungoides: Clinicopathological and Prognostic Features of 45 Cases. French Study Group of Cutaneious Lymphomas. Blood (2000) 95(7):2212-8.

76. Benner MF, Jansen PM, Vermeer MH, Willemze R. Prognostic Factors in Transformed Mycosis Fungoides: A Retrospective Analysis of 100 Cases. Blood (2012) 119(7):1643-9. doi: 10.1182/blood-2011-08-376319

77. Lansigan F, Horwitz SM, Pinter-Brown LC, Carson KR, Shustov AR, Rosen ST, et al. Outcomes of Patients With Transformed Mycosis Fungoides: Analysis From a Prospective Multicenter US Cohort Study. Clin Lymphoma Myeloma Leuk (2020) 20(11):744-8. doi: 10.1016/j.clml.2020.05.001

78. Hodak E, Amitay-Laish I, Atzmony L, Prag-Naveh H, Yanichkin N, Barzilai A, et al. New Insights Into Folliculotropic Mycosis Fungoides (FMF): A Single-Center Experience. J Am Acad Dermatol (2016) 75(2):347-55. doi: 10.1016/j.jaad.2016.03.009
79. Kosaka A, Ohkuri T, Ikeura M, Kohanbash G, Okada H. Transgene-Derived Overexpression of miR-17-92 in CD8+ T-Cells Confers Enhanced Cytotoxic Activity. Biochem Biophys Res Commun (2015) 458(3):549-54. doi: 10.1016/ j.bbrc.2015.02.003

80. Ohno M, Ohkuri T, Kosaka A, Tanahashi K, June $\mathrm{CH}$, Natsume A, et al. Expression of miR-17-92 Enhances Anti-Tumor Activity of T-Cells Transduced With the Anti-EGFRvIII Chimeric Antigen Receptor in Mice Bearing Human GBM Xenografts. J Immunother Cancer (2013) 1:21. doi: 10.1186/2051-1426-1-21

81. Cyrenne BM, Lewis JM, Weed JG, Carlson KR, Mirza FN, Foss FM, et al. Synergy of BCL2 and Histone Deacetylase Inhibition Against Leukemic Cells From Cutaneous T-Cell Lymphoma Patients. Blood (2017) 130(19):2073-83. doi: 10.1182/blood-2017-06-792150

82. Wollina U, Dummer R, Brockmeyer NH, Konrad H, Busch JO, Kaatz M, et al. Multicenter Study of Pegylated Liposomal Doxorubicin in Patients With Cutaneous T-Cell Lymphoma. Cancer (2003) 98(5):993-1001. doi: 10.1002/ cncr.11593

83. Takahashi RU, Prieto-Vila M, Kohama I, Ochiya T. Development of miRNABased Therapeutic Approaches for Cancer Patients. Cancer Sci (2019) 110 (4):1140-7. doi: $10.1111 /$ cas.13965

84. Travaglino A, Varricchio S, Pace M, Russo D, Picardi M, Baldo A, et al. Borrelia Burgdorferi in Primary Cutaneous Lymphomas: A Systematic Review and Meta-Analysis. J Dtsch Dermatol Ges (2020) 18(12):1379-84. doi: 10.1111/ddg.14289

85. Tothova SM, Bonin S, Trevisan G, Stanta G. Mycosis Fungoides: Is It a Borrelia Burgdorferi-Associated Disease? Br J Cancer (2006) 94(6):879-83. doi: $10.1038 /$ sj.bjc. 6602997

86. Abrams JT, Balin BJ, Vonderheid EC. Association Between Sézary T CellActivating Factor, Chlamydia Pneumoniae, and Cutaneous T Cell Lymphoma. Ann N Y Acad Sci (2001) 941:69-85. doi: 10.1111/j.17496632.2001.tb03712.x

87. Liu K, Pan X, Peng X, Zhang C, Li H, Guan X, et al. Associations of High Expression of miR-106b-5p Detected From FFPE Sample With Poor Prognosis of RCC Patients. Pathol Res Pract (2019) 215(6):152391. doi: 10.1016/j.prp.2019.03.019

88. Querfeld C, Foss FM, Pinter-Brown LC, Porcu P, William BM, Pacheco T, et al. Phase 1 Study of the Safety and Efficacy of MRG-106, a Synthetic Inhibitor of microRNA-155, in CTCL Patients. Blood (2017) 1(1):820. doi: 10.1182/blood.V130.Suppl_1.820.820

Conflict of Interest: The authors declare that the research was conducted in the absence of any commercial or financial relationships that could be construed as a potential conflict of interest.

Publisher's Note: All claims expressed in this article are solely those of the authors and do not necessarily represent those of their affiliated organizations, or those of the publisher, the editors and the reviewers. Any product that may be evaluated in this article, or claim that may be made by its manufacturer, is not guaranteed or endorsed by the publisher.

Copyright $\odot 2021$ Wen, Xie and Wang. This is an open-access article distributed under the terms of the Creative Commons Attribution License (CC BY). The use, distribution or reproduction in other forums is permitted, provided the original author(s) and the copyright owner(s) are credited and that the original publication in this journal is cited, in accordance with accepted academic practice. No use, distribution or reproduction is permitted which does not comply with these terms. 